\title{
Successful Treatment of Recurrent Renal Artery Stenosis in a Patient with Moyamoya Disease by Aorto-Renal Bypass with Autogenous Vein Graft
}

\author{
Hyunmin $\mathrm{Ko}^{1}$ and Seung-Kee $\mathrm{Min}^{2}$ \\ ${ }^{1}$ Department of Surgery, Kyung Hee University Hospital, Seoul, ${ }^{2}$ Division of Vascular Surgery, Department of Surgery, Seoul National \\ University Hospital, Seoul, Korea
}

A 20-year-old male patient was transferred to our vascular clinic with recurrent renal artery stenosis (RAS). At 12 years of age, he had visited a neurosurgery clinic with transient left hemiparesis and dizziness after eating ra- myun. Magnetic resonance imaging revealed bilateral internal carotid artery (ICA) stenosis and small infarctions in the right temporal to occipital lobe compatible with Moyamoya disease (MMD). Transfemoral carotid angiography

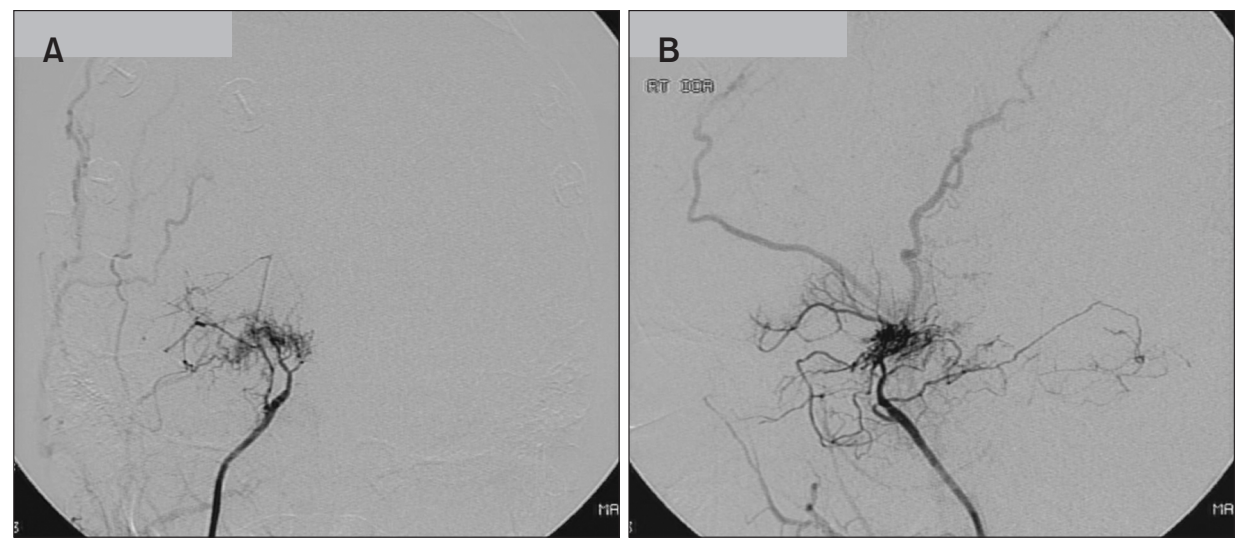

Fig. 1. Transfemoral carotid angiography showed bilateral internal carotid artery (ICA) stenosis. (A) Left ICA, (B) right ICA.
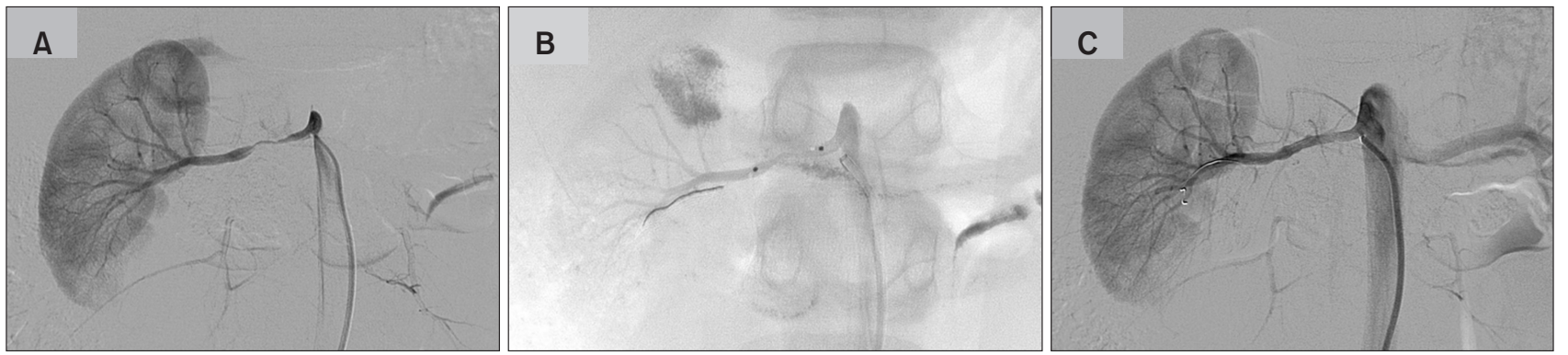

Fig. 2. (A) Digital subtraction angiography showed severe stenosis of the right renal artery. (B) Balloon angioplasty was performed. (C) Completion angiography showed a well-dilated renal artery with good renal perfusion.

Received December 17, 2021, Accepted December 21, 2021, Published on January 17, 2022

Corresponding author: Seung-Kee Min, Division of Vascular Surgery, Rm 5313, Biomedical Research Institute, Seoul National University Hospital, 101 Daehak-ro, Jongno-gu, Seoul 03080, Korea

Tel: 82-2-2072-0297, Fax: 82-2-766-3975, E-mail: skminmd@snuh.org, http://orcid.org/0000-0002-1433-2562

Copyright (c) 2022 The Korean Society for Vascular Surgery

This is an Open Access article distributed under the terms of the Creative Commons Attribution Non-Commercial License (http://creativecommons.org/licenses/by-nc/4.0) which permits unrestricted non-commercial use, distribution, and reproduction in any medium, provided the original work is properly cited.

Cite this article; Vasc Specialist Int 2022. https://doi.org/10.5758/vsi.210079 
confirmed both ICA stenosis (Fig. 1) and right RAS (Fig. 2). Neurosurgeons performed four indirect bypass (encephaloduro-arterio-synangiosis) operations to improve brain perfusion. For the RAS, balloon angioplasty was performed successfully (Fig. 3). However, when he was 19 years of age, the disease progressed to severe right RAS and newonset left RAS. Balloon angioplasty of the left renal artery was performed. However, 6 months later, left RAS redeveloped (Fig. 4). The right kidney was severely atrophic, and a quantitative dimercaptosuccinic acid (DMSA) scan showed no function of the right atrophic kidney (Fig. 5). Therefore, to treat the renovascular hypertension (RVHT) and preserve the renal mass of the single left kidney, we performed a right nephrectomy and aorto-left renal bypass graft with a reversed saphenous vein. Aspirin $100 \mathrm{mg}$ was administered, and the graft remained patent at the 2-year follow-up.

MMD is a progressive cerebrovascular disorder showing steno-occlusion of the bilateral internal carotid arteries that results in smoke-like vascular networks at the base of the brain. Choi et al. [1] reported 8.3\% cases of RVHT among

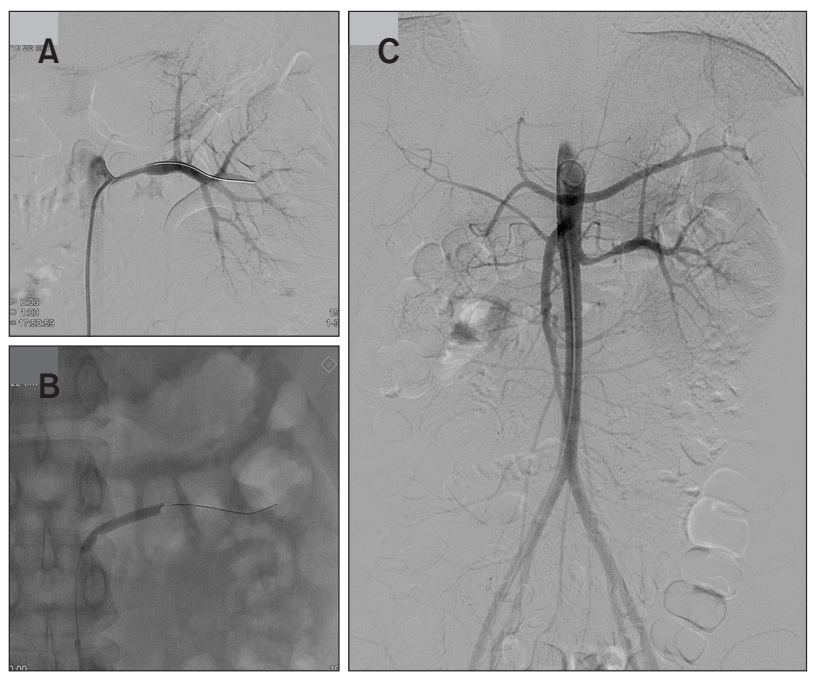

Fig. 3. (A) Digital subtraction angiography showed severe stenosis of left renal artery. (B) Balloon angioplasty was performed. (C) Completion angiography showed mild residual stenosis with good renal perfusion.
72 MMD patients and concluded that RVHT may be more commonly associated with MMD than previously believed. Therefore, careful blood pressure monitoring should be performed in MMD and diagnostic procedures for RVHT should be followed in hypertensive patients with MMD. Experience with pediatric MMD cases in Seoul National University Hospital was published elsewhere [2,3]. This case highlights the progressive nature of RAS when refractory to balloon angioplasty in MMD. Regular careful imaging follow-up is important in RVHT in MMD.

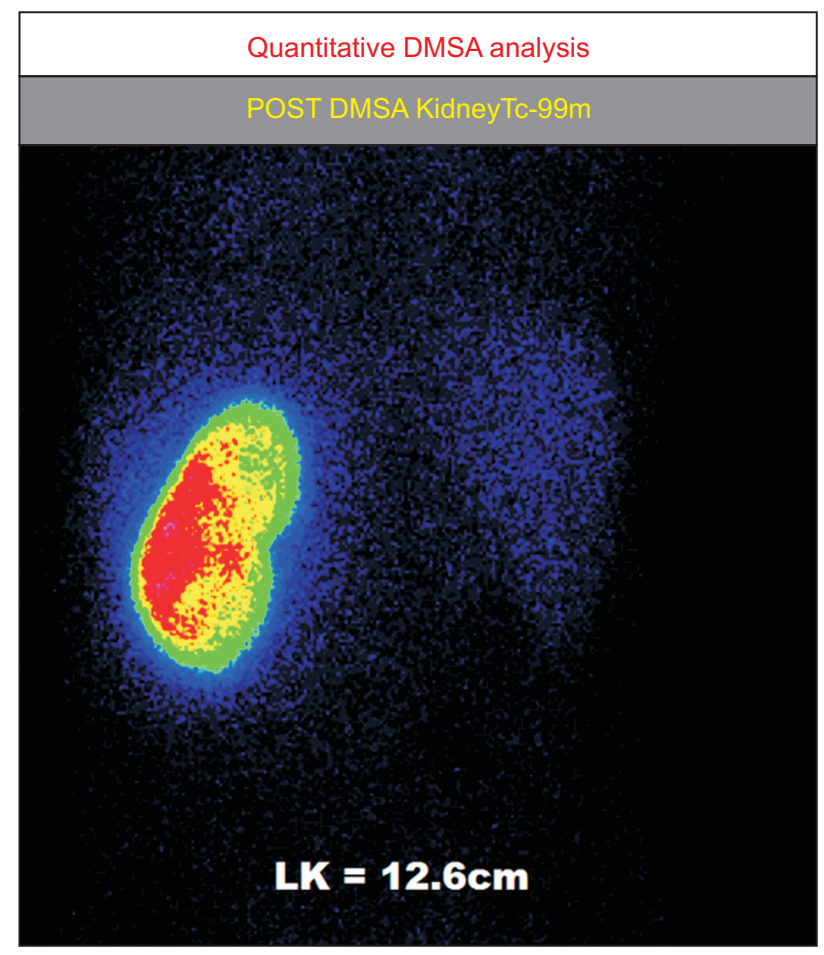

\begin{tabular}{|l|c|c|c|}
\hline & Left kidney & Right kidney & Both kidney \\
\hline Absolute uptake & $44.7 \%$ & $0.0 \%$ & $44.7 \%$ \\
\hline Posterior relative uptake & $100.0 \%$ & $0.0 \%$ & $100.0 \%$ \\
\hline Mean relative uptake & $100.0 \%$ & $0.0 \%$ & $100.0 \%$ \\
\hline
\end{tabular}

Fig. 5. Quantitative dimercaptosuccinic acid (DMSA) scan showed no function of the right atrophic kidney. LK, left kidney.
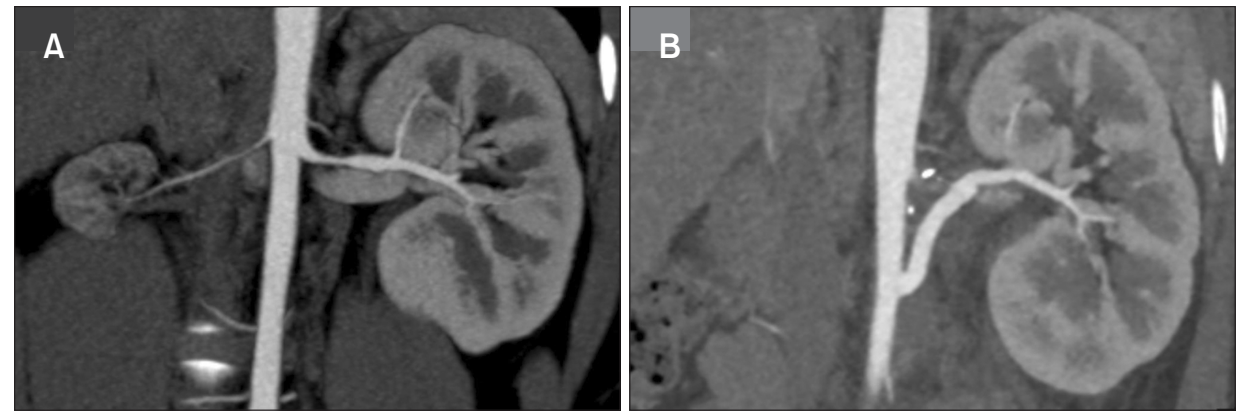

Fig. 4. (A) Computed tomography angiography (CTA) revealed severe stenosis of the right renal artery with an atrophic kidney and recurrent stenosis of the left renal artery. (B) CTA showed the patent left aortorenal bypass graft with good renal perfusion. 
1) Choi $Y$, Kang BC, Kim KJ, Cheong HI, Hwang YS, Wang KC, et al. Renovascular hypertension in children with moyamoya disease. J Pediatr 1997;131:258-263.
2) Choi JW, Chong S, Phi JH, Lee JY, Kim HS, Chae JH, et al. Postoperative symptomatic cerebral infarction in pediatric moyamoya disease: risk factors and clinical outcome. World Neurosurg 2020;136:e158-e164.
3) Min SK, Cho S, Kim HY, Kim SJ. Pediatric vascular surgery review with a 30-year-experience in a tertiary referral center. Vasc Specialist Int 2017;33:47-54. 